\title{
Large Eddy Simulations of large wind-turbine arrays in the atmospheric boundary layer
}

\author{
Johan Meyers* \\ Department of Mechanical Engineering, Katholieke Universiteit Leuven, \\ Celestijnenlaan 300A - bus 2421, B3001 Leuven, Belgium \\ Charles Meneveau ${ }^{\dagger}$ \\ Department of Mechanical Engineering and Center for Environmental and Applied Fluid Mechanics, \\ Johns Hopkins University, 3400 North Charles Street, Baltimore MD 21218, USA
}

Large Eddy Simulations (LES) of arrays of wind turbines in the atmospheric turbulent boundary layer with many turbines often require simplified models for the effects of individual turbines, in order to avoid having to use very fine grid spacings near the individual (moving) turbine blades. This goal can be accomplished using the actuator disk model. This approach, however, raises several issues when implemented in the context of LES. In particular, the question is raised of which reference velocity should be used when parameterizing the induced forces: instantaneous versus time averaged, undisturbed velocity or the local one. Also, one may consider including tangential forces to represent the angular momentum in the wakes. In this paper we present several arguments to make the appropriate choices, and illustrate the effects of these choices in LES of two wind turbine arrays, one with an aligned and one with a staggered arrangement. Predicted power outputs, as well as features of the predicted flow fields are analyzed.

\section{Nomenclature}

$\begin{array}{ll}C_{P}, C_{P}^{\prime} & \text { power coefficients } \\ C_{T}, C_{T}^{\prime} & \text { thrust coefficients } \\ \mathbf{f}_{\mathbf{t}} & \text { wind-turbine forces } \\ \widehat{\mathbf{f}}_{\mathbf{t}}, \widehat{\mathbf{f}} & \text { wind-turbine forces on coarse LES grid } \\ \mathbf{h} & \text { grid spacing }\left(h_{1}, h_{2}, h_{3}\right) \\ H & \text { height of the atmospheric boundary layer } \\ \widetilde{\mathbf{u}} & \left.\text { LES velocity field (components } \widetilde{u}_{1}, \widetilde{u}_{2}, \widetilde{u}_{3}\right) \\ V & \text { disk-average axial velocity at the turbine } \\ \bar{V} & \text { time-averaged disk-average axial velocity at the turbine } \\ \mathbf{x} & \text { LES coordinate frame } \\ G r e e k & \\ \Delta & \text { filter width for filtering of } \mathbf{f} \\ \Omega & \text { angular velocity }\end{array}$

\section{Introduction}

In order for wind power to become a significant contributor to the worldwide overall energy portfolio, wind turbines will need to be deployed in very large wind farms. Envisioning such large-scale implementations calls for advancements in our understanding of the detailed interactions between wind turbines and the

\footnotetext{
*Email: johan.meyers@mech.kuleuven.be

†Email: meneveau@jhu.edu
} 
atmospheric surface layer. Increased understanding is required to improve siting of wind-farms, to optimize the spatial distribution of wind turbines inside an array, to better quantify unsteady loading and failure predictions, ${ }^{1}$ as well as to parameterize their effects in very large-scale simulations at regional ${ }^{2}$ or global $^{3}$ scales. Computer simulations are playing an increasingly important role for addressing both applied design and fundamental research questions. In the wind energy field, the traditional simulation tool is Reynolds Averaged Navier-Stokes (RANS) (see e.g. Ref. 4,5).

The relevant phenomena occur at many length and time scales, with atmospheric motions ranging from kilometer scale, down to turbulence at scales below $1 \mathrm{~mm}$ in the boundary layers surrounding the individual wind-turbine blades. The structure of the atmospheric boundary layer (ABL) evolves over a daily cycle, ${ }^{6}$ with thermal forcing during the day causing strong buoyant conditions while surface cooling at nightime typically generates stably stratified conditions. Wind-turbines must operate in this variable environment and the various conditions must be well understood to better predict temporal variability of the highly intermittent wind resource. Capturing all scales of this highly complex process both in space and time during a single simulation is not possible. In order to capture the large-scale structures of the turbulent atmospheric boundary layer covering a significant number of wind turbines, domain sizes of several kilometers in horizontal extent are needed. By necessity, wind turbines must therefore be represented using approximations. The most popular concept is that of an "actuator disk". Initial advances in using Large Eddy Simulation (LES) in conjunction with the "actuator disk" concept have been made recently by Jimenez et al. ${ }^{7}$ In this method the wind turbine is represented by a force acting on the grid-points where the rotor disk is located. The method is efficient due its lower requirements of grid resolution and coarser allowed time-stepping as compared to having to resolve detailed flow surrounding rotating blades. A more refined approach relying on forces distributed along rotating lines ("actuator line method") $)^{8}$ has also been proposed, but it too requires significantly smaller mesh sizes than what is practical for LES with domain sizes that cover large extensions. In the present work, several open aspects of the "actuator disk" methodology are addressed and implemented in the context of LES. Ultimately, our goal is to use the methodology developed in conjunction with an optimization method to improve power-extraction under realistic constraints.

In the "actuator disk" approach, ${ }^{9}$ the axial force added on grid-points where the rotor disk is located is set equal to

$$
F_{x}=-\frac{1}{2} C_{T} \rho V_{\infty}^{2} A_{c}
$$

where $C_{T}$ is the thrust coefficient and $A_{c}$ is the cell frontal area, and $V_{\infty}$ is the undisturbed velocity that would have existed on the rotor disk if no wind turbine was present. In deciding what values of $C_{T}$ to use, it matters a great deal how exactly the other variables in the equation are interpreted. For instance, is $V_{0}$ the mean undisturbed velocity or a local instantaneous value? How should one determine this undisturbed velocity in cases when wind-turbines influence each other in large arrays? In prior works using the actuator disk approach (e.g. Ref. 7), a value of $C_{T}=0.75$ has been used in conjunction with a fixed velocity that was specified from a log-law at hub height. However, if wind turbines are in each other's wakes it is not clear which velocity scale should be used. In particular, one would prefer to use a local velocity at the rotor plane. Also, how to determine the power output from such implementations is not immediately clear. Moreover, the angular momentum in the wake may influence interactions between the wind turbine and the atmospheric boundary layer and so a model should attempt to reproduce the angular momentum. This can be accomplished in the "actuator disk" framework by distributing tangential forces on grid points. The aim of the work presented in this paper is to formulate and choose among various options, implement them in LES of wind-turbine arrays and document the effects of various options. We concentrate on an 'infinite' array of turbines, representing the effect of 'very large' wind farms on the ABL. This allows the use of simulation domains with periodic boundary conditions in streamwise and spanwise directions, as is common practice in LES of the atmospheric boundary layer, since it allows for a very efficient and accurate discretization of the governing equations based on Fourier spectral method.

The current paper is organized as follows. First, in Secion II, the computational model is introduced: in IIA the LES equations are presented, next in IIB the implementation of the turbine forces is discussed, and finally in IIC further details of the computational set up are presented. Subsequently, simulation results are discussed in Section III, and conclusions are formulated in Section IV. 


\section{Computational model}

\section{A. Governing equations}

Large Eddy Simulation is based on the filtered Navier-Stokes equations, ${ }^{10,11}$ which for an incompressible and neutral (non-bouyant) flow reads

$$
\frac{\partial \widetilde{\mathbf{u}}}{\partial t}+\widetilde{\mathbf{u}} \cdot \nabla \widetilde{\mathbf{u}}=-\frac{1}{\rho} \nabla \widetilde{p}-\nabla \boldsymbol{\tau}_{s g s}+\widehat{\mathbf{f}}, \quad \nabla \cdot \widetilde{\mathbf{u}}=0
$$

$\widetilde{\mathbf{u}}$ is the velocity field (with components $\widetilde{u}_{i}, i=1 \cdots 3$ ), $\widetilde{p}$ the pressure, $\boldsymbol{\tau}_{s g s}$ the subgrid-scale tensor, and $\widehat{\mathbf{f}}$ additional forcing terms, which in the current paper correspond to the actuator-disk forces (see below) per unit mass. Molecular viscous stresses are omitted from the equations, since we focuss on LES of the atmospheric boundary layer, in which the Reynolds number is very high and effects of viscosity on the resolved scales is negligible. The effect of turbulence motions at scales smaller than the grid-size on the resolved field $\widetilde{\mathbf{u}}$ is modeled using the Smagorinksy subgrid-scale closure

$$
\boldsymbol{\tau}_{s g s}=-2 \ell^{2}(2 \mathbf{S}: \mathbf{S})^{1 / 2} \mathbf{S},
$$

with $\mathbf{S}=\left(\nabla \widetilde{\mathbf{u}}+(\nabla \widetilde{\mathbf{u}})^{T}\right) / 2$ the strain-rate tensor, and $\ell$ is the characteristic length-scale for the eddy-viscosity. At this stage we use a non-dynamic version of the Smagorinsky model, which requires damping functions to account for effects near the ground, i.e. the length-scale is obtained ${ }^{10}$ from

$$
\frac{1}{\ell^{n}}=\frac{1}{\left[\kappa\left(z+z_{0}\right)\right]^{n}}+\frac{1}{\left(C_{s} h\right)^{n}}
$$

with $z_{0}$ the roughness of the ground surface, $\kappa$ the von Kármán constant (we use $\kappa=0.4$ ), and $h=$ $\left(h_{1} h_{2} h_{3}\right)^{1 / 3}$ the mesh spacing. For the current work, we employ standard parameters according to $C_{s}=0.13$, $n=3$.

High Reynolds number LES modeling of atmospheric boundary layers over rough surfaces also requires modeling of the wall stress. Again, we follow standard approaches ${ }^{10,12}$ and determine the wall stress by using the velocity vector at the first grid-point above the ground surface (at $\left.z_{1}=h_{3} / 2\right)$ according to:

$$
\tau_{i, 3}^{w}=-\left[\frac{\kappa}{\log \left(z_{1} / z_{0}\right)}\right]^{2}\left(\widetilde{u}_{1}\left(x, y, z_{1}\right)^{2}+\widetilde{u}_{2}\left(x, y, z_{1}\right)^{2}\right)^{1 / 2} \widetilde{u}_{i}\left(x, y, z_{1}\right),
$$

where $i=1,2$ in the horizontal direction.

The flow is artificially forced in stream wise direction with a volume forcing corresponding to a constant pressure gradient $\partial p / \partial x_{1}=1$ (also corresponding to a friction velocity $u_{\tau}=1$ ). This approach is common practice in large-eddy simulations of boundary layers and channel flows. It allows the use of periodic boundary conditions in the streamwise direction and periodic boundary conditions are also used for the spanwise direction. The governing equations (2) are then discretized in streamwise $x$ and spanwise $z$ directions based on a pseudo-spectral Fourier method, and dealiasing is performed using the $2 / 3^{\text {rd }}$ dealiasing rule. Fourier transforms are implemented using the FFTW library. ${ }^{13}$ The wall-normal direction is discretized using a fourth-order energy-conserving staggered finite-volume discretization, ${ }^{14}$ i.e. the locations of wall-normal velocities $\bar{v}$ are shifted in the wall-normal direction by half a cell compared to $\bar{p}, \bar{u}$, and $\bar{w}$. Continuity is retained by solving a Poisson equation for the pressure, using a direct solver, which is based on a Cholesky factorization. The time integration is performed by a classical four-stage fourth-order accurate Runge-Kutta time integration. ${ }^{15}$ Time steps are restricted by setting both the convective and viscous CFL number to 0.4. Extensive tests show that the code without wind-turbines reproduces known mean and second-order statistics as usually found in LES of atmospheric boundary layers.

\section{B. Wind-farm model and forces}

For sake of convenience, we introduce some geometrical definitions related to the plane of the turbine rotor. Consider $\mathbf{y}=\left(y_{1}, y_{2}\right)$ a coordinate system in the turbine rotor plane, with origin in the rotor center, $y_{1}$ the horizontal, and $y_{2}$ the vertical direction. Further, take $\mathbf{f}_{\mathbf{t}}(\mathbf{y})$ the distribution of forces in that plane. In the LES coordinate frame the rotor plane is given by coordinates $\mathbf{x}_{\mathbf{t}}$ which satisfy $\mathbf{e}_{\perp} \cdot\left(\mathbf{x}_{\mathbf{t}}-\mathbf{x}_{\mathbf{0}}\right)=0$ with $\mathbf{x}_{\mathbf{0}}$ the 
coordinate of the rotor center, and $\mathbf{e}_{\perp}$ a unit vector perpendicular to the rotor plane. Moreover, a relation $\mathbf{y}=\mathbf{y}\left(\mathbf{x}_{\mathbf{t}}-\mathbf{x}_{\mathbf{0}}\right)$ exists that allows to map the local plane coordinates to those of the LES.

In order to project the actuator disk force $\mathbf{f}_{\mathbf{t}}$ (to be specified later) onto the LES grid, we propose to use a three-dimensional gaussian convolution filter. In general, such a filter applied on a tensor $\mathbf{g}(\mathbf{x})$ is defined by

$$
\widehat{\mathbf{g}}(\mathbf{x})=\iiint G\left(\mathbf{x}-\mathbf{x}^{\prime}\right) \mathbf{g}\left(\mathbf{x}^{\prime}\right) \mathrm{d}^{3} \mathbf{x}^{\prime}
$$

with $\mathbf{x}^{\prime}$ an auxiliary integration coordinate, and $G(\mathbf{x})$ the filter kernel, which corresponds for a gaussian filter to $G(\mathbf{x})=\left[6 /\left(\pi \Delta^{2}\right)\right]^{3 / 2} \exp \left(-6\|\mathbf{x}\|^{2} / \Delta^{2}\right)$. Applying Eq. (6) to the turbine forces $\mathbf{f}_{\mathbf{t}}$ leads to

$$
\widehat{\mathbf{f}}_{\mathbf{t}}(\mathbf{x})=\iiint G\left(\mathbf{x}-\mathbf{x}^{\prime}\right) \delta\left(\mathbf{e}_{\perp} \cdot\left(\mathbf{x}^{\prime}-\mathbf{x}_{\mathbf{0}}\right)\right) \mathbf{f}_{\mathbf{t}}\left(\mathbf{y}\left(\mathbf{x}^{\prime}-\mathbf{x}_{\mathbf{0}}\right)\right) \mathrm{d}^{3} \mathbf{x}^{\prime},
$$

where $\delta(x)$ is the Dirac function (a similar smoothing approach was used for actuator-line representations in Ref. 16). In order to properly align the filtered forces $\widehat{\mathbf{f}}_{\mathbf{t}}$ with the LES coordinate frame $\mathbf{x}$, an additional rotation operation is needed, which is expressed as

$$
\widehat{\mathbf{f}}(\mathbf{x})=\mathbf{Q} \cdot \widehat{\mathbf{f}}_{\mathbf{t}}(\mathbf{x})
$$

where $\mathbf{Q}$ is a rotation matrix, accounting for possible different orientations between the $\mathbf{y}$, and $\mathbf{x}$ coordinate frames, and $\widehat{\mathbf{f}}$ are the properly oriented forces entering into Eq. (2). In the current work, all turbines are aligned perpendicular to $x_{1}$, such that $\mathbf{Q}$ is an identity matrix, and $\widehat{\mathbf{f}}=\widehat{\mathbf{f}}_{\mathbf{t}}$.

In the special case that one of the force components $f_{t, i}$ is constant over the rotor disk (as we will presume, e.g. for the thrust force, see below), Eq. (7) further simplifies to $\widehat{f}_{t, i}(\mathbf{x})=f_{t, i} \mathscr{R}(\mathbf{x})$, with

$$
\mathscr{R}(\mathbf{x})=\iiint G\left(\mathbf{x}-\mathbf{x}^{\prime}\right) \delta\left(\mathbf{e}_{\perp} \cdot\left(\mathbf{x}^{\prime}-\mathbf{x}_{\mathbf{0}}\right)\right) \mathrm{H}\left(R-\left\|\mathbf{y}\left(\mathbf{x}^{\prime}-\mathbf{x}_{\mathbf{0}}\right)\right\|\right) \mathrm{d}^{3} \mathbf{x}^{\prime},
$$

the geometrical representation of the rotor disk smoothed out into the LES domain using a gaussian filter. Above, $\mathrm{H}(x)$ is the Heaviside function and $R$ the disk radius. In order to determine the force, we will require the disk-averaged velocity $\mathbf{V}$. In order to obtain $\mathbf{V}$ at the rotor disk we propose to use the geometrical rotor footprint $\mathscr{R}(\mathbf{x})$ as a weighting function for the averaging, i.e.

$$
\mathbf{V}=\iiint \mathscr{R}(\mathbf{x}) \mathbf{u}(\mathbf{x}) \mathrm{d}^{3} \mathbf{x} .
$$

Hence, we define the axial disk-averaged velocity $V$ as $V=\mathbf{V} \cdot \mathbf{e}_{\perp}$.

In order to implement Eqs. (7) and (9) we do not advocate to integrate these equations analytically, which can lead to rather awkward expressions when the turbine forces $\mathbf{f}_{\mathbf{t}}$ are complex functions themselves. Instead, we represent $\mathbf{f}_{\mathbf{t}}$ on a very fine two-dimensional turbine grid, and perform the integration in Eqs. (7) and (9) numerically at each coarse-grid point of the LES grid. Moreover, since the gaussian filter kernel $G\left(\mathbf{x}-\mathbf{x}^{\prime}\right)$ drops very fast to zero for $\left\|\mathbf{x}-\mathbf{x}^{\prime}\right\| \gg \Delta$, the integration can be restricted to an area close to $\mathbf{x}$, which makes this a very efficient approach.

As discussed in $\S 1$, to simulate arrays with interacting wind turbines, it is more natural to use a velocity scale that is local at the rotor disk. Hence, we define the axial disk-averaged velocity $V$ as $V=\mathbf{V} \cdot \mathbf{e}_{\perp}$. When simulating wind turbines in a turbulent unsteady flow, there is the additional question of time averaging. As will be explained later, the appropriate scale is the time and disk-averaged velocity. Then, the forces that model the wind turbine are modeled according to

$$
\begin{aligned}
f_{x} & =-\frac{1}{2} C_{T}^{\prime} \bar{V}^{2}, \\
f_{\theta} & =\frac{1}{2} C_{P}^{\prime} \bar{V}^{2} \frac{V}{\Omega r} .
\end{aligned}
$$

Here, $f_{x}$, and $f_{\theta}$ are the axial and tangential forces (per unit surface and per unit density) constituting the turbine forces $\mathbf{f}_{\mathbf{t}}$ (we drop the subscript 't' for convenience), $V$ is the axial velocity averaged over the rotor disk, and $\bar{V}$ the time-averaged value of $V$. Further $C_{T}^{\prime}$ and $C_{P}^{\prime}$ are respectively thrust and power coefficients. They are defined using the velocity at the disk, and hence differ from the usual thrust and power coefficients 
$\left(C_{T}\right.$ and $\left.C_{P}\right)$ commonly employed in the literature, which are based on the undisturbed upstream velocity $V_{\infty}$ at hub height. In the following, we will briefly outline the basis for Eqs. (11) and (12) by reviewing some well known elements of blade element momentum theory, and the relation between $C_{T}^{\prime}, C_{P}^{\prime}$ and $C_{T}$, $C_{P}$ is further elaborated.

Blade element momentum theory is a methodology for wind-turbine design, which combines Glauert's actuator disk theory with a description of lift and drag forces on the turbine blades. A detailed overview can, e.g., be found in Ref. 9. Firstly, we recall definitions of the axial induction factor $a$, and the tangential flow induction factor $a^{\prime}$ :

$$
V \equiv V_{\infty}(1-a), \quad \omega \equiv 2 \Omega a^{\prime},
$$

with $\omega$ the angular velocity of the wake, and $\Omega$ the angular velocity of the turbine rotor.

We will now base the ensuing arguments on two assumptions. First of all, we presume a turbine which is rotating at an angular velocity which is optimally adapted to the time-averaged incoming velocity $\bar{V}$, and we presume that the tip-speed ratio, which we define here as $\lambda=\Omega R / \bar{V}$ is constant (note that, similar to $C_{T}^{\prime}, C_{P}^{\prime}$, we prefer to define $\lambda$ based on the local blade velocity, instead of $V_{\infty}$ ). Secondly, we assume that the ratio of lift and drag coefficient of the blades remains constant (this presumes an active pitch control of the turbine, which adapts the angle of the blades to changes in incoming velocity).

The tangential force per unit length $\delta F_{\theta}$ on the turbine blade at radius $r$, responsible for the torque on the turbine, can now be written as

$$
\delta F_{\theta}(r)=\frac{1}{2} c_{L} \rho V_{r e l}^{2} n c \cos \beta-\frac{1}{2} c_{D} \rho V_{r e l}^{2} n c \sin \beta,
$$

with $c_{L}$, and $c_{D}$ the lift and drag coefficient of the blade profile (at radius $r$ ), $V_{\text {rel }}$ incoming velocity relative to the rotating blades (at radius $r$ ), $n$ the number of blades, $c(r)$ the chord length (at radius $r$ ), and $\beta$ the angle between the relative velocity, and the turbine axis. Accounting for the blade speed $\Omega r$ at radius $r$, and the induced swirl by the blades (which adds a tangential velocity to the velocity on the turbine blade equal to $\Omega r a^{\prime}$ ), the relative velocity and angle $\beta$ correspond with ${ }^{9}$

$$
V_{\text {rel }}=\left[V^{2}+\left(\Omega r\left(1+a^{\prime}\right)\right)^{2}\right]^{1 / 2}, \quad \cos \beta=V /\left[V^{2}+\left(\Omega r\left(1+a^{\prime}\right)\right)^{2}\right]^{1 / 2}
$$

To obtain Eq. (12), Eq. (14) can be used to write

$$
P=\Omega T=\Omega \int_{0}^{R} r \delta F_{\theta}(r) \mathrm{d} r
$$

To perform this integration, the knowledge of the chord length, lift and drag coefficients, and twist of the blade as function of the radius is in principle required. In order to circumvent such a detailed description in the current approach we presume blades which are optimally designed for the tip-speed $\lambda=\Omega R / \bar{V}$. It is well established that a turbine is optimally designed when ${ }^{9}$

$$
a^{\prime}=\frac{a}{(1-a)(\Omega r)^{2}} .
$$

Moreover, to achieve this, the turbine blades (i.e., lift coefficient, chord length, and blade twist as function of radius) should be designed such that the tangential force $\delta F_{\theta}$ is independent of the radius. Further elaboration of this idea (neglecting drag, etc., cf. Ref. 9), leads to following optimality conditions, which will be further used

$$
\frac{1}{2} c_{L} \rho V_{\text {rel, dsgn }}^{2} \cos \beta_{d s g n} n c=4 \pi \rho \frac{V_{d s g n}^{3}}{\Omega} \frac{a}{1-a} .
$$

Depending on a selected design velocity $V_{d s g n}$ and angular velocity, from which follow $V_{\text {rel,dsgn }}$, and $\beta_{d s g n}$, this relation provides a geometrical description of the blade chord. As discussse above, we take $V_{d s g n}=\bar{V}$. Using Eq. (15), Equation (18) is reformulated into

$$
\frac{1}{2} c_{L} \rho c_{L} \rho \bar{V}\left[\bar{V}^{2}+\left(\Omega r\left(1+a^{\prime}\right)\right)^{2}\right]^{1 / 2} n c=4 \pi \rho \frac{\bar{V}^{3}}{\Omega} \frac{a}{1-a} .
$$


Further inserting Eq. (19) in Eq.(12) and using Eqs. (15) leads to

$$
\begin{aligned}
\delta F_{\theta} & =4 \pi \rho \frac{\bar{V}^{2} V}{\Omega} \frac{a}{1-a} \frac{\left[V^{2}+\left(\Omega r\left(1+a^{\prime}\right)\right)^{2}\right]^{1 / 2}}{\left[\bar{V}^{2}+\left(\Omega r\left(1+a^{\prime}\right)\right)^{2}\right]^{1 / 2}}\left(1-\frac{c_{D}}{c_{L}} \frac{\Omega r\left(1+a^{\prime}\right)}{V}\right), \\
& =4 \pi \rho \frac{\bar{V}^{2} V}{\Omega} \frac{a}{1-a} \frac{\left[V^{2} / \bar{V}^{2}+\left(\lambda \mu\left(1+a^{\prime}\right)\right)^{2}\right]^{1 / 2}}{\left[1+\left(\lambda \mu\left(1+a^{\prime}\right)\right)^{2}\right]^{1 / 2}}\left(1-\frac{c_{D}}{c_{L}} \frac{\lambda \mu\left(1+a^{\prime}\right)}{V / \bar{V}}\right)
\end{aligned}
$$

In Eq. (21) we further introduced normalized radius $\mu=r / R$ and the tip-speed ratio $\lambda$ defined before.

Equation (17) can now be simplified by recognizing that $V / \bar{V}$ is fluctuating around one. For $\lambda \mu \gg 1$, this fluctuation becomes negligible in the term $\left[V^{2} / \bar{V}^{2}+\left(\lambda \mu\left(1+a^{\prime}\right)\right)^{2}\right]$. Since typically, $5<\lambda<10$, this is the case for most of the turbine blade outside the hub region. Hence,

$$
\delta F_{\theta} \approx 4 \pi \rho \frac{\bar{V}^{2} V}{\Omega} \frac{a}{1-a}\left(1-\frac{c_{D}}{c_{L}} \lambda \mu\left[1+\frac{a}{(1-a) \lambda^{2} \mu^{2}}\right]\right)
$$

and inserting in Eq. (16) and integrating leads to

$$
\delta P=\frac{1}{2} C_{P}^{\prime} \rho \bar{V}^{2} V \delta A, \quad \text { with } \quad C_{P}^{\prime}=\frac{4 a}{1-a}\left(1-\frac{c_{D}}{c_{L}} \lambda\left[\frac{2}{3}+\frac{2 a}{(1-a) \lambda^{2}}\right]\right)
$$

and $\delta A=2 \pi r \delta r$. A similar derivation for the axial force, which is not further elaborated here, leads to

$$
\delta F_{x}=-\frac{1}{2} C_{T}^{\prime} \rho \bar{V}^{2} \delta A, \text { with } C_{T}^{\prime}=\frac{4 a}{1-a}\left(1+\frac{c_{D}}{c_{L}} \frac{2}{\lambda}\right) .
$$

Finally, Eq. (11) and (12) are recovered as $\delta F_{x} /(2 \pi r \rho)$ and $\delta F_{\theta} /(2 \pi r \rho)$

The most relevant question is which values to use for $C_{T}^{\prime}$ and $C_{P}^{\prime}$ in order to specify the axial and tangential forces in the simulation. For sake of clarity, we neglected effects related to the tip-loses in the derivations of Eq. (23) and (24). Instead of including them here (e.g., by using Prandtl's tip-loss factor), we will use following straightforward relations between $C_{T}^{\prime}, C_{P}^{\prime}$, and $C_{T} C_{P}$, i.e.

$$
C_{P}=(1-a)^{3} C_{P}^{\prime} \quad \text { and } \quad C_{T}=(1-a)^{2} C_{T}^{\prime} .
$$

Using a set of known empirical values, i.e. we take $C_{T} \approx 0.75$ and $a=1 / 4$, and $C_{P} \approx 0.34$, so we obtain $C_{T}^{\prime}=4 / 3$, and $C_{P}^{\prime} \approx 0.8$.

\section{Computational set-up}

An overview of the computational set-up is given in Tables 1 and 2. Simulations are run using $N_{x} \times N_{y} \times N_{z}=$ $64 \times 160 \times 60$ grid points. Simulations are initialized with a pre-computed turbulent boundary layer without wind turbines. Subsequently, a sufficiently long initialization run is performed for the atmospheric boundary layer to reach a new equilibrium. In total 36 turbines are equally spaced in the computational domain. More details are given in the tables.

Four cases are simulated and compared: (i) turbines aligned without tangential forcing, (ii) with tangential forcing; and (iii) turbine staggered without, and (iv) with tangential forcing. A detail of the grid for the staggered case is presented in Figure 1

\section{Results and discussion}

By comparing the results of wind-farm simulations with and without tangential forces, we find that the effect of adding tangential forces on the mean stream-wise velocity and the average power extracted from the atmospheric boundary layer is negligible. The most important differences between cases with and without swirl may be seen by looking at stream-wise vorticity plots (not shown here), or span-wise and normal components of the velocity. In Figure 2, the normal velocity is displayed in the turbine wake for the aligned case, and a clear difference is visible between the two cases. Currently, we have been running our simulations 
Table 1. General computational set-up

\begin{tabular}{ll}
\hline \hline Reference length & height of the ABL, $H \equiv 1$ \\
Reference length & friction velocity, $u_{\tau} \equiv 1$ \\
Domain height & $0.9 H$ \\
Domain width & $2.4 H$ \\
Domain length & $4.2 H$ \\
Wall roughness & $z_{0} / H=10^{-4}$ \\
Model constants & $C_{s}=0.13, n=3$ \\
Grid resolution & $N_{x} \times N_{y} \times N_{z}=64 \times 160 \times 60$ \\
CFL number & 0.4 \\
Averaging time & $140 H / u_{\tau}$
\end{tabular}

Table 2. Wind-farm set-up

\begin{tabular}{ll}
\hline \hline Hub height & $0.1 H$ \\
Turbine radius & $R=0.05 H$ \\
Turbine arrangement & inline or staggered \\
Turbine spacing & $\Delta X=0.7 H=7 D$ \\
& $\Delta Y=0.4 H=4 D$ \\
Angular velocity & $\Omega=1.5 \times 10^{3} u_{\tau} / H$ \\
$C_{T}^{\prime} ; C_{P}^{\prime}$ & $1.333 ; 0.8$ \\
$C_{T} ; C_{P}($ taking $a=1 / 4)$ & $0.75 ; 0.45$ \\
Turbine-force mesh & $100 \times 100$ (per turbine) \\
Filter width $\Delta$ & $1.5 h$
\end{tabular}

at a moderate $C_{P}=0.34$ and relatively high tip-speed ratio. Whether the effect of tangential forces on the mean stream-wise velocity becomes more prominent with a higher $C_{P}$ and lower angular velocity, is subject of ongoing simulations.

In Figure 3 the streamwise time-averaged velocity is displayed for the aligned and staggered simulations with tangential forces. The figures are a zoom on $1 / 9$ th of the computational domain $\left(L_{y} / 3 \times L_{z} / 3\right)$. This not only allows a better view of the differences in the wake structures around the turbine, but allows an additional averaging of results over all 9 subregions for better statistical convergence. It is appreciated from Figure 3 that there are distinct differences in the flow pattern between the aligned and the staggered farm. In particular, the aligned geometric arrangement allows for 'high-speed channels' between the turbine rows, and an elongated streamwise low-speed region along the aligned turbine axis. In the staggered case, the turbine wakes have more time to recover before they hit the next turbine, providing a slightly higher velocity at the turbines. No high-speed stream-wise channels are visible. Further, the staggered geometric arrangement appears more effective in dragging the atmospheric boundary layer, which results in an overall lower average velocity.

Finally, we evaluate the power extracted from the ABL by the turbines in Figure 4. For this we use $P=C_{T}^{\prime} \bar{V}^{2} V A$. Note that the usable power which can be converted to electricity is a factor $C_{P}^{\prime} / C_{T}^{\prime}$ lower (the difference corresponds with losses related to drag, which are dissipated into subgrid-scale energy and heat). In Figure 4(a) the average power extracted per turbine is displayed as function of time for the aligned case. Here the effect of the changeable turbulent ABL conditions on the power output is very much visible. In this figure, the standard deviation on the power extraction over the 36 turbine in the domain is also displayed, illustrating the large spread among turbines in instantaneous power extracted. In Figure 4(b) the power extraction of the aligned and staggered case are compared by looking at a running average over the 


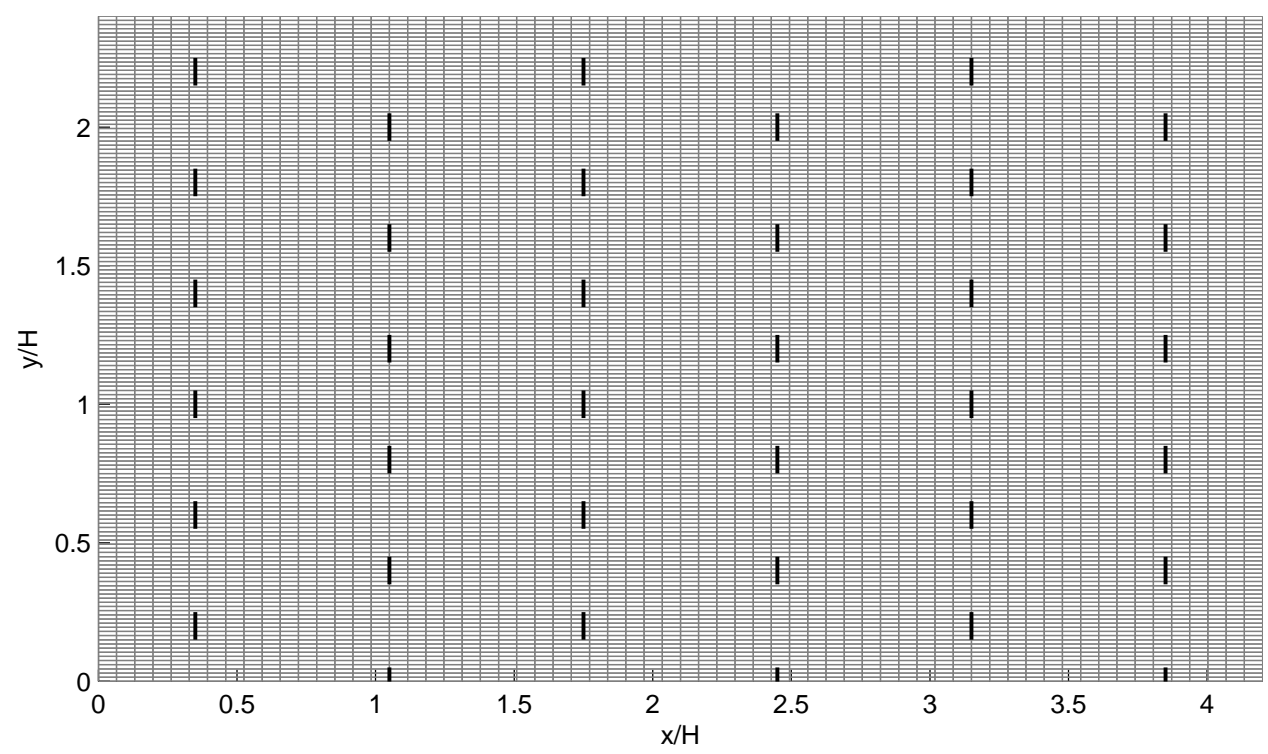

Figure 1. Computational grid and placement of the turbines for the staggered case. XY-plane.

simulation time window. Here, it becomes clear that the staggered farm has an overall power extraction which is about $5 \%$ higher than the aligned farm.

\section{Conclusions}

The current paper presented a study of large-scale wind turbine arrays in the atmospheric boundary layer using large-eddy simulations. We used an "actuator disk" approach for the modeling of the turbines. We focussed on the correct scaling of the forces with time-averaged and instantaneous velocity, and showed that the thrust forces scale with $\bar{V}^{2}$, while tangential forces scale with $\bar{V}^{2} V / \Omega r$.

Several simulations were performed, with and without tangential forces, and both representing turbines in aligned arrays and in staggered arrays. We found that the addition of tangential forces has its largest effect on the normal and spanwise velocity. However, the effect on the mean streamwise velocity and the total extracted power is negligible. When comparing the aligned farm with the staggered farm, it was shown that the latter extracts about $5 \%$ more power than the former. This can be related to distinct differences in flow features between the two farms.

To fully understand the interaction of large wind farms with the atmospheric boundary layer, and to optimize wind-farm siting, various issues need further investigation. This includes, e.g, the asymptotic equilibrium behavior of the atmospheric boundary layer in the presence of large wind farms: a set of simulations focussing on effective roughness heights and kinetic energy fluxes is reported in Ref. 17. The effect large 'finite' farms on the ABL, and effects of variability of wind speed and directions are also of interest for further study. With respect to optimization of turbine siting, reducing the variability of power output (next to increasing the total power output), is an interesting topic for future research.

\section{Acknowledgments}

JM acknowledges funding from the Research Foundation - Flanders (FWO - Vlaanderen). CM thanks the National Science Foundation for research funding (CBET-0730922). 

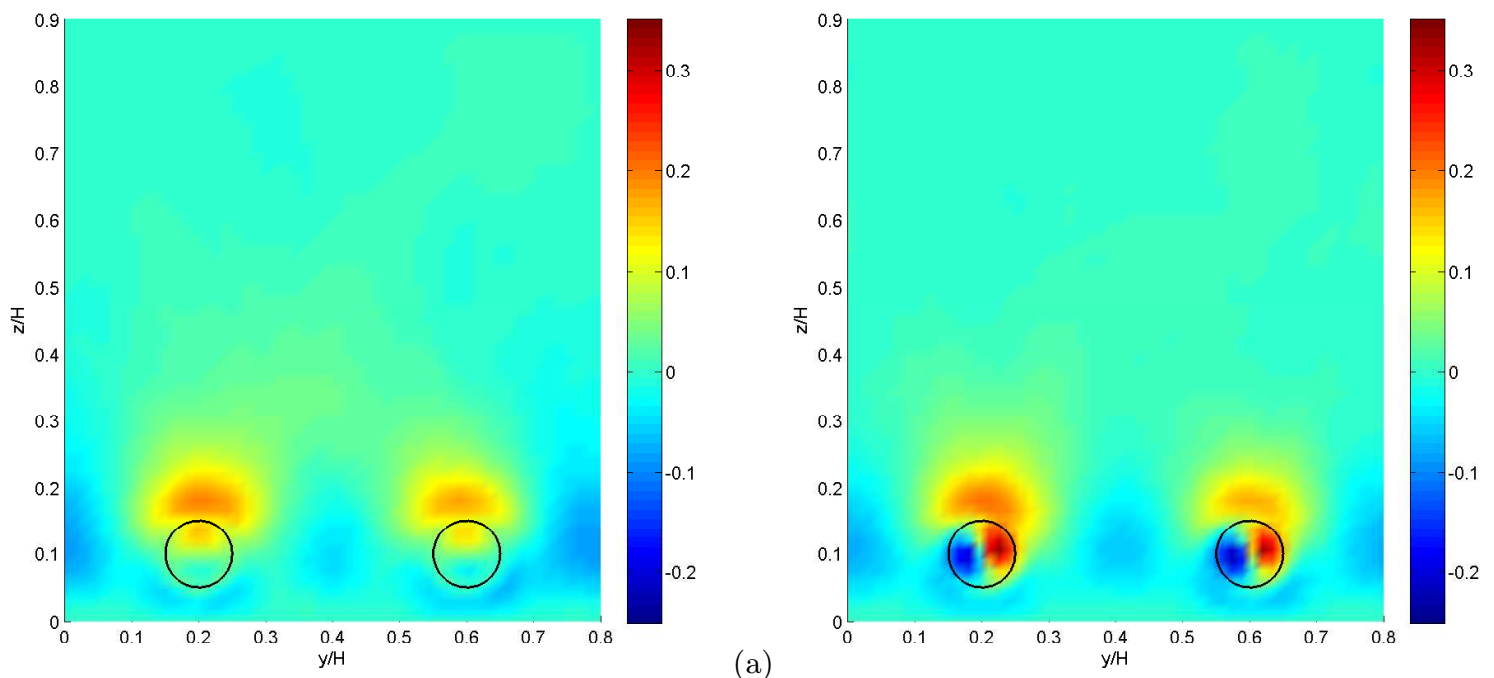

(a)

Figure 2. YZ-cut of the normal velocity $\left\langle\widetilde{u}_{3}\right\rangle$ in a plane $0.15 H$ downstream of the turbine. Only $1 / 3$ of the $Y$-domain is shown. Aligned case without tangential forces (a) and with tangential forces (b).

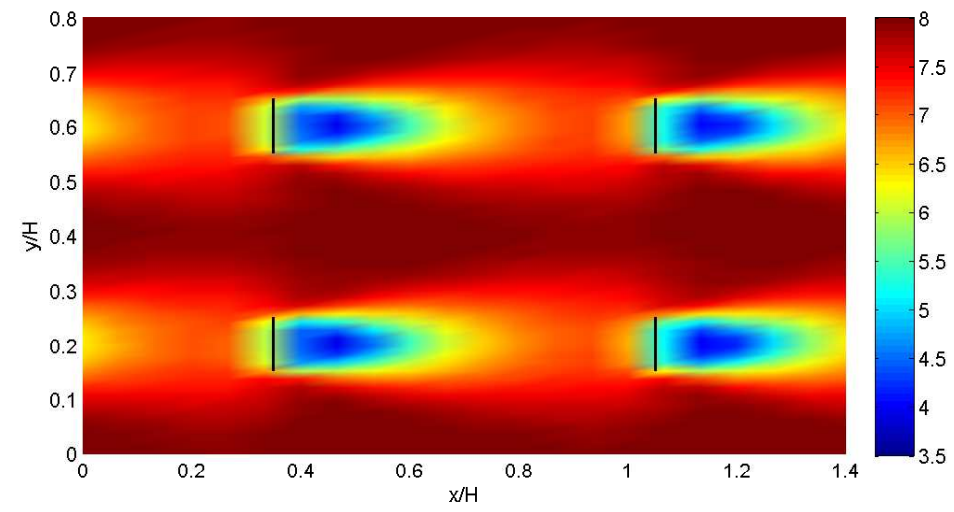

(a)

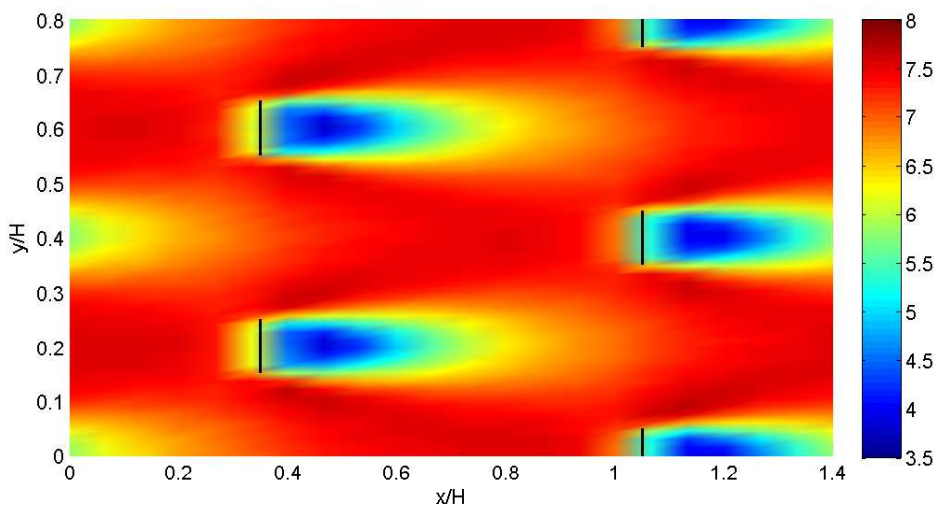

(b)

Figure 3. XY-cut of the streamwise averaged velocity $\left\langle\widetilde{u}_{1}\right\rangle$ at turbine-hub height $(z=0.1 H)$. (a) aligned case ; (b) staggered case. Only 1/9th of the domain is shown $\left(L_{y} / 3 \times L_{z} / 3\right)$, and results of all 9 regions are averaged for better statistical convergence. 


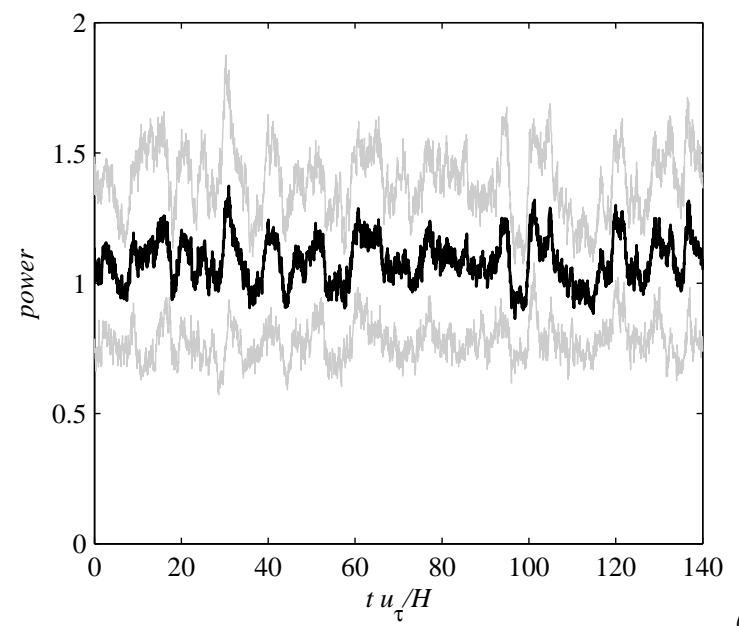

(a)

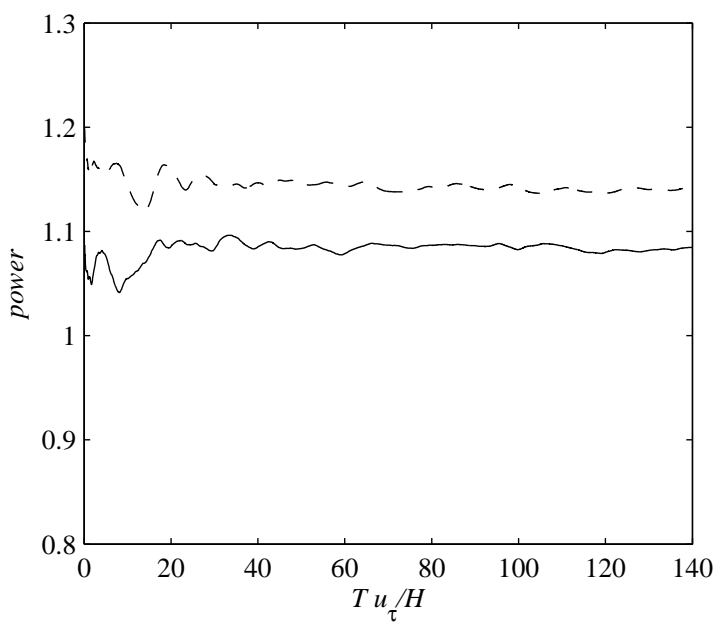

(b)

Figure 4. (a) Time history of average power extracted from the boundary layer for the aligned case. (black): average power extracted per turbine; (gray) \pm standard deviation based on 36 turbines. (b) Running time average of the power extraction per turbine as function of the averaging period T. (--): staggered; (-): aligned case.

\section{References}

${ }^{1}$ Frandsen, S. and Th ogersen, M., "Integrated fatigue loading for wind turbines in wind farms by combining ambient turbulence and wakes," Wind. Eng., Vol. 23, 1999, pp. 327-340.

${ }^{2}$ Baidya-Roy, S., Pacala, S., and Walko, R., "Can large scale wind famrs affect local meteorology?" J. Geophys. Res., Vol. 109, 2004, pp. D19101.

${ }^{3}$ Keith, D., DeCarolis, J., Denkenberger, D., Lenschow, D., Malyshev, S., Pacala, S., and Rasch, P. J., "The influence of large-scale wind power on global climate," Proceeding of National Academy of Sci., Vol. 101, 2004, pp. 16115-16120.

${ }^{4}$ Sorensen, N. and Michelsen, J., "Drag prediction for blades at high angle of attack using CFD," J. of Solar Energy Eng., Vol. 126, 2002, pp. 1011-1016.

${ }^{5}$ Johansen, J., Sorensen, N., and Schreck, S., "Detached-Eddy Simulation of Flow around the NREL Phase-VI RotorD," Wind Energy, Vol. 5, 2003, pp. 185-197.

${ }^{6}$ Kumar, V., J, K., Parlange, M., and Meneveau, C., "Large-Eddy Simulation of a diurnal cycle in the turbulent Atmospheric Boundary Layer: Atmospheric stability and scaling issue," Water Res. Research, Vol. 42, 2006, pp. W06D09.

${ }^{7}$ Jimenez, A., Crespo, A., Migoya, E., and Garcia, J., "Advances in large-eddy simulation of a wind turbine wake," J. of Physics: Conference Series, Vol. 75, 2007, pp. 012041.

${ }^{8}$ Mikkelsen, R., Sorensen, J., Oye, S., and Troldborg, N., "Analysis of power enhancement for a row of wind turbines using the actuator line technique," J. of Physics: Conference Series, Vol. 75, 2007, pp. 012044.

${ }^{9}$ Burton, T., Sharpe, D., Jenkins, N., and Bossanyi, E., Wind Energy Handbook, John Wiley \& Sons, Ltd., (2001).

${ }^{10}$ Mason, P., "Large-eddy simulation: a critical review of the technique," Q.J.R. Meteorol. Soc., Vol. 120, (1994), pp. 1.

${ }^{11}$ Piomelli, U., "Large eddy simulation: achievements and challenges," Progr. in Aerosp Sci., Vol. 35, (1999), pp. 335-362.

${ }^{12}$ Moeng, C.-H., "A large-eddy simulation model for the study of planetary boundary-layer turbulence," J. Atmos. Sci., Vol. 6, (1984), pp. 2311-2330.

${ }^{13}$ Frigo, M. and Johnson, S. G., "The Design and Implementation of FFTW3," Proceedings of the IEEE, Vol. 93, No. 2, 2005, pp. 216-231, Special issue on "Program Generation, Optimization, and Platform Adaptation".

${ }^{14}$ Verstappen, R. W. C. P. and Veldman, A. E. P., "Symmetry-preserving discretization of turbulent flow," Journal of Computational Physics, Vol. 187, 2003, pp. 343-368.

${ }^{15}$ Hirsch, C., Fundamentals of Numerical Discretization, Vol. 1 of Numerical Computation of Internal and External Flows, John Willey \& Sons, 1988.

${ }^{16}$ Sorensen, J. and Shen, W., "Numerical Modeling of Wind Turbine Wakes," J. Fluids Eng., Vol. 124, 2002, pp. 393-399.

${ }^{17}$ Calaf, M., Meneveau, C., and Meyers, J., "Large Eddy Simulation study of fully developed wind-turbine array boundary layers," Phys. Fluids, in review. 